\title{
An Efficient Equi-semi-join Algorithm for Distributed Architectures
}

\author{
M. Bamha and G. Hains \\ LIFO, Université d'Orléans, B.P. 6759, 45067 Orléans Cedex 2, France \\ \{bamha, ghains\}@lifo.univ-orleans.fr
}

\begin{abstract}
Semi-joins is the most used technique to optimize the treatment of complex relational queries on distributed architectures. However the overcost related to semi-joins computation can be very high due to data skew and to the high cost of communication in distributed architectures. In this paper we present a parallel equi-semi-join algorithm for shared nothing machines. The performance of this algorithm is analyzed using the BSP cost model and is proved to have asymptotic optimal complexity and perfect load balancing even for highly skewed data. This guarantees unlimited scalability in all situations for this key algorithm.
\end{abstract}

\section{Introduction}

Equi-join is an expensive and frequently used operation whose parallelization is highly desirable. The equi-join of two tables or relations $R$ and $S$ on attribute $A$ of $R$ and attribute $B$ of $S$ is the relation $T$, written $R \bowtie S$, containing the pairs of tuples from $R$ and $S$ for which $R . A=S . B$. The equi-semi-join of $S$ by $R$ is the relation $S \ltimes R$ composed of the tuples of $S$ which occur in the equi-join of $R$ and $S$. Equi-semi-join reduces the size of relations to be joined and satisfies $R \bowtie S=R \bowtie(S \ltimes R)=(R \ltimes S) \bowtie(S \ltimes R)$. The equi-semi-join is generally called semi-join, and throughout the paper, semi-join refers to equi-semi-join.

The semi-join is useful in relational databases for reducing the time for processing queries involving join operations, by means of selecting only relevant data and thereby reducing the size of operand relations. Semi-join is also used to reduce the amount of data transferred over the network and therefore the communication costs in distributed architectures [10,8]. However efficient semi-join is very sensitive to the problem of data skew. Data skew can have a disastrous effect on performance $[3,2,1,8,7,6,11,5]$ due to the high costs of communications and synchronizations in distributed architectures $[2,1,10]$.

Many algorithms have been proposed to handle data skew for join and semijoin operations $[8,7,6,10]$. Such algorithms are not efficient for many reasons:

- the presented algorithms are not scalable (and thus cannot guarantee linear speedup) because their routing decisions are generally performed by a coordinator processor while the other processors are idle,

- they cannot avoid load imbalance because they base their routing decisions on incomplete or statistical information, 
- they cannot solve data skew problem because data redistribution is generally based on hashing data into buckets and hashing is known to be inefficient.

In this paper, we present a new parallel semi-join algorithm for shared nothing machines. This algorithm guarantees a perfect balancing of the load of the different processors during all the stages of the semi-join computation because the data redistribution is carried out jointly by all processors. Each processor deals with the data associated with a subset of the join attribute values (organized as histograms) not necessarily its "own" values. Our algorithm uses histogram's redistribution to avoid imbalance during semi-join parallel processing. Its performance is analyzed using Bulk-synchronous parallel (BSP) cost model [9] which allows us to guarantee that histogram management has a negligible cost when compared to the efficiency gains it provides to reduce the communication cost and to avoid load imbalance between processors.

\section{Histograms and Semi-joins Processing: A New Approach}

In this section, we present a new approach to compute semi-joins on shared nothing machines. This approach is insensitive to data skew, and is based on an efficient technique for histograms computation, allowing to reduce the net communication costs to a minimum.

Before carrying out the description of the semi-join algorithm, we present some results related to histograms computations which we will use thereafter. An upper bound of the global BSP cost is also given for each step.

We first assume that relation $R$ (resp. $S$ ) is partitioned among processors by horizontal fragmentation and the fragments $R_{i}$ for $i=1, . ., p$ are almost of the same size, i.e. $\left|R_{i}\right| \simeq \frac{|R|}{p}$ where $p$ is the number of processors.

In the rest of this paper, we use the following notation for each relation $T \in\{R, S\}:$

$-T_{i}$ denotes the fragment of relation $T$ placed on processor $i$,

- Hist $(T)$ denotes the histogram of relation $T$ with respect to the join attribute value, i.e. a list of pairs $\left(v, n_{v}\right)$ where $n_{v} \neq 0$ is the number of tuples of relation $T$ having the value $v$ for the join attribute,

- $\operatorname{Hist}\left(T_{i}\right)$ denotes the histogram of fragment $T_{i}$,

- $\operatorname{Hist}_{i}(T)$ is processor $i$ 's fragment of the histogram of $T$,

- $\|T\|$ denotes the number of tuples of relation $T$, and

- $|T|$ denotes the size (expressed in bytes or number of pages) of relation $T$.

It will be assumed in the remainder of the article that, for a relation $T,\|T\|$ is sufficiently large with respect to $p$ and in the rest of the paper, $g$ refers to BSP communication's parameter and $l$ the cost for a barrier of synchronization [9].

Proposition 1. Let Hist $\left(R_{i}\right)_{i=1, \ldots, p}$ be the histograms, according to some attribute $A$, of the fragments $R_{i}$ of a relation $R$. If $\mathcal{H}$ is a hash function allowing 
to distribute in a balanced way the distinct values of the attribute $A$ among the $p$ processors, then the fragments $\operatorname{Hist}_{i}(R)_{i=1, \ldots, p}$ can be computed in time:

$$
O\left(\min \left(g *|H i s t(R)|+\|\operatorname{Hist}(R)\|, g * \frac{|R|}{p}+\frac{\|R\|}{p}\right)+l\right) .
$$

Proof: The proof proceeds according to frequency distribution of the attribute $A$ of the relation $R$.

$\mathbf{1}^{\text {st }}$ case: All the values of the attribute $A$ appear in the relation $R$ with a frequency equal to 1 , i.e. the number of tuples of the relation $R$ is equal to the number of distinct values of the attribute $A$ (and thus $\|H i s t(R)\|=\|R\|$ ).

In this case, the application of the hash function $\mathcal{H}$, distributes the tuples of relation $R$ (and thus the histogram of $R$ ) evenly on the various processors. Consequently, the redistribution of $\operatorname{Hist}\left(R_{i}\right)$, using the function $\mathcal{H}$, is done in time:

$$
O\left(g * \frac{|H i s t(R)|}{p}+\frac{\|H i s t(R)\|}{p}+l\right) \leq O\left(g * \frac{|R|}{p}+\frac{\|R\|}{p}+l\right) .
$$

The term $g * \frac{|H i s t(R)|}{p}$ is due to the fact that each processor receives a fragment of size about equal to $\frac{|H i s t(R)|}{p}, g$ is BSP communication parameter. The term $\frac{\|\operatorname{Hist}(R)\|}{p}$ is time necessary to form $\operatorname{Hist}_{i}(R)_{i=1, . ., p}$ starting from the blocks received by each processor, during the redistribution of the local histograms $\operatorname{Hist}\left(R_{i}\right)_{i=1, \ldots, p}$ and $l$ is the cost of a barrier of synchronization.

Inequality (1) is generally true, owing to the fact that $|H i s t(R)| \leq|R|$ since the histogram contains only pairs of the form $\left(v, n_{v}\right)$ where $v$ are distinct values of attribute $\mathrm{A}$ of $R$ and $n_{v}$ the corresponding frequencies. So the histograms creation can be carried out in time:

$$
\begin{aligned}
& O\left(\min \left(g * \frac{|H i s t(R)|}{p}+\frac{\|\operatorname{Hist}(R)\|}{p}, g * \frac{|R|}{p}+\frac{\|R\|}{p}\right)+l\right) \\
& \leq O\left(\min \left(g *|\operatorname{Hist}(R)|+\|\operatorname{Hist}(R)\|, g * \frac{|R|}{p}+\frac{\|R\|}{p}\right)+l\right) .
\end{aligned}
$$

$\mathbf{2}^{\text {nd }}$ case: All the values of the attribute $A$ appear in relation $R$ with a frequency lower than $p$. In this case the relation $R$ can be partitioned into $p$ relations $R^{1}$, $R^{2}, \ldots, R^{p}$ so that the frequency of each value $v$ in a relation $R^{j}$ is exactly equal to 1 . By applying the results of the $1^{\text {st }}$ case to each relation $R^{j}$, we deduce that the creation of the fragments $\operatorname{Hist}_{i}\left(R^{j}\right)$ of a relation $R^{j}$ for $j=1, \ldots, p$ requires at most:

$$
O\left(\min \left(g * \frac{\left|\operatorname{Hist}\left(R^{j}\right)\right|}{p}+\frac{\left\|\operatorname{Hist}\left(R^{j}\right)\right\|}{p}, g * \frac{\left|R^{j}\right|}{p}+\frac{\left\|R^{j}\right\|}{p}\right)+l\right),
$$

and thus the creation of the fragments $\operatorname{Hist}_{i}(R)$ of relation $R$ requires:

$$
\begin{array}{r}
O\left(\sum_{j=1, . . p} \min \left(g * \frac{\left|H i s t\left(R^{j}\right)\right|}{p}+\frac{\left\|H i s t\left(R^{j}\right)\right\|}{p}, g * \frac{\left|R^{j}\right|}{p}+\frac{\left\|R^{j}\right\|}{p}\right)+l\right) \\
\leq O\left(\min \left(g *|\operatorname{Hist}(R)|+\|\operatorname{Hist}(R)\|, g * \frac{|R|}{p}+\frac{\|R\|}{p}\right)+l\right),
\end{array}
$$


because the fragment $\operatorname{Hist}_{i}(R)$ can be created starting from the fragments $\operatorname{Hist}_{i}\left(R^{j}\right)_{j=1, . ., p}$ by a simple fusion of the histograms $\operatorname{Hist}_{i}\left(R^{j}\right)_{1 \leq j \leq p}$ in time:

$$
O\left(\sum_{j=1}^{p} \frac{\| \text { Hst }_{i}\left(R^{j}\right) \|}{p}\right) \leq O\left(\max _{i, j}\left(\left\|\operatorname{Hist}_{i}\left(R^{j}\right)\right\|\right)\right) \leq O(\|H i s t(R)\|),
$$

and that $\sum_{i} \min \left(a_{i}, b_{i}\right) \leq \min \left(\sum_{i} a_{i}, \sum_{i} b_{i}\right)$. We thus combine the $p$ phases of communication in only one (the term is not multiplied by $p$ ).

The distribution of the relation $R$ into $p$ relations is carried out only to show the validity of the result. In practice, if a value $v$ appears in $R_{i}$ with a frequency $k$, the application of the hash function to the histogram sends the pair $(v, k)$ to the processor destination in only one message and not in $k$ messages of the form $(v, 1)$. This reduces the amount of data exchanged between the processors.

$3^{\text {rd }}$ case: All the values of the attribute $A$ appear in the relation $R$ with frequencies higher than $p$. In this case, for a given value $v$, the tuples associated with $v$ can be distributed on several processors and in the worst case distributed on $p$ processors. Thus the application of the hash function, $\mathcal{H}$, to the histograms $\operatorname{Hist}\left(R_{i}\right)_{i=1, . ., p}$, sends pairs of the form $\left(v, k_{i}\right)_{i=1, . ., p}$ to the same processor.

As each processor deals with a block of size $\frac{\|H i s t(R)\|}{p}$, it receives at most $p * \frac{|H i s t(R)|}{p}=|H i s t(R)|$ data from the other processors, and the cost of the redistribution of the histograms $\operatorname{Hist}\left(R_{i}\right)$ is about:

$$
O(g *|H i s t(R)|+l) \leq O\left(g * \frac{|R|}{p}+l\right)
$$

Inequality (4) holds because all the values of attribute $A$ appear in $R$ with a frequency higher than $p$ and thus: $\|\operatorname{Hist}(R)\| \leq \frac{\|R\|}{p} \quad$ and $\quad|\operatorname{Hist}(R)| \leq \frac{|R|}{p}$.

$\|H i s t(R)\|$ is the time necessary to form, $H_{i s t}(R)$, on processor $i$, starting from the fragments of $H i s t\left(R_{j}\right)_{j=1, \ldots, p}$ received after the redistribution phase using the hash function $\mathcal{H}$. And thus the creation of the histograms $\operatorname{Hist}_{i}(R)$ requires:

$$
O\left(\min \left(g *|\operatorname{Hist}(R)|+\|\operatorname{Hist}(R)\|, g * \frac{|R|}{p}+\frac{\|R\|}{p}\right)+l\right) .
$$

$4^{\text {th }}$ case: the general case follows immediately from the three preceding cases. We partition relation $R$ into two disjoint sub-relations $R^{\prime}$ and $R^{\prime \prime}$ so that the tuples of the relation $R^{\prime}$ (resp. $R^{\prime \prime}$ ) are associated with frequencies lower (resp. higher) than $p$ for the attribute $A$. Thus, using the first two cases, the histogram $R^{\prime}$ can be carried out in time:

$$
O\left(\min \left(g *\left|\operatorname{Hist}\left(R^{\prime}\right)\right|+\left\|\operatorname{Hist}\left(R^{\prime}\right)\right\|, g * \frac{\left|R^{\prime}\right|}{p}+\frac{\left\|R^{\prime}\right\|}{p}\right)+l\right),
$$

and the creation of the histogram of $R^{\prime \prime}$, (cf. 3rd case), in time:

$$
O\left(\min \left(g *\left|H i s t\left(R^{\prime \prime}\right)\right|+\left\|\operatorname{Hist}\left(R^{\prime \prime}\right)\right\|, g * \frac{\left|R^{\prime \prime}\right|}{p}+\frac{\left\|R^{\prime \prime}\right\|}{p}\right)+l\right)
$$


By making the sum of the terms 5 and 6 , we show that the creation of the histograms $\mathrm{Hist}_{i}(R)$ requires at most:

$$
O\left(\min \left(g *|H i s t(R)|+\|H i s t(R)\|, g * \frac{|R|}{p}+\frac{\|R\|}{p}\right)+l\right) .
$$

This is due to the fact that $\operatorname{Hist}(R)=\operatorname{Hist}\left(R^{\prime}\right) \cup H i s t\left(R^{\prime \prime}\right)$ and that $\min (a, b)+$ $\min (c, d) \leq \min (a+c, b+d)$, which proves the required result.

Previous parallel algorithms have used hashing functions to redistribute the relations themselves, not just their histograms. This can lead to imbalance and disastrous performance.

Remark 1. In practice, the imbalance of the data related to the use of the hash functions can be due to:

- a bad choice of the hash function used. This imbalance can be avoided by using the hashing techniques presented in the literature making it possible to distribute evenly the values of the join attribute with a very high probability [4]. In our algorithm, the hash functions we use may be data dependent and stored in the database index. Otherwise, for a given set of $n$ distinct values we can find, in time of $0(n)$, a function which redistributes evenly the $n$ values over $p$ buckets.

- an intrinsic data imbalance which appears when some values of the join attribute appear more frequently than others. By definition a hash function maps tuples having the same join attribute values to the same processor. There is no way for a clever hash function to avoid load imbalance that results from these repeated values [5]. But this case cannot arise here owing to the fact that histograms contain only distinct values of the join attribute and the hashing functions we use are always applied to histograms.

In the following proposition, we present a method allowing to compute the semi-joins efficiently starting from the histograms. We show thereafter that this technique of semi-joins computation combined with the methods of histograms creation guarantees an asymptotic optimal cost for the semi-joins computation.

Proposition 2. Let Hist $\left(R_{i}\right)_{i=1, \ldots, p}$ (resp. Hist $\left.\left(S_{i}\right)_{i=1, \ldots, p}\right)$ be the histograms, according to some attribute $A$, of sub-relation $R_{i}$ (resp. $S_{i}$ ) of $R$ (resp. $S$ ). If $\mathcal{H}$ is a hash function allowing to distribute in a balanced way, the distinct values of the attribute $A$ among the p processors, then the two semi-joins, $R \ltimes S$ and $S \ltimes R$, can be computed in time:

$$
\begin{aligned}
\text { Time }_{s j}= & O\left(\min \left(g *|\operatorname{Hist}(R)|+\|\operatorname{Hist}(R)\|, g * \frac{|R|}{p}+\frac{\|R\|}{p}\right)+\max _{i=1, \ldots, p}\left\|R_{i}\right\|\right. \\
& \left.+\min \left(g *|\operatorname{Hist}(S)|+\|\operatorname{Hist}(S)\|, g * \frac{|S|}{p}+\frac{\|S\|}{p}\right)+\max _{i=1, \ldots, p}\left\|S_{i}\right\|+l\right) .
\end{aligned}
$$


Proof: To perform the semi-joins in at most the above time, the semi-joins computation, $R \ltimes S$ and $S \ltimes R$, is done in five stages.

a. A stage of creation of $H_{i s t}(R)_{i=1, \ldots, p}\left(\operatorname{resp} . H_{i s t}(S)_{i=1, \ldots, p}\right)$ of $R$ (resp. $\left.S\right)$ : The fragments $H_{i s t}(R)_{i=1, . ., p}$ creation is carried out by hashing the local histograms $H i s t\left(R_{i}\right)_{i=1, \ldots, p}$ using the hash function $\mathcal{H}$. On each processor $i$, the application of the function $\mathcal{H}$ distributes $\operatorname{Hist}\left(R_{i}\right)$ into $p$ buckets $\operatorname{Hist}^{j}\left(R_{i}\right)_{i=1, \ldots, p}$ where $\operatorname{Hist}^{j}\left(R_{i}\right)$ is the fragment of $\operatorname{Hist}\left(R_{i}\right)$ which will be sent to processor $j$. By using Proposition 1, we have: $\left|\cup_{i} \operatorname{Hist}^{j}\left(R_{i}\right)\right| \leq \min \left(|\operatorname{Hist}(R)|, \frac{|R|}{p}\right)$.

The fragments $H_{i s t}{ }^{j}\left(R_{i}\right)_{i=1, . ., p}$ are then gathered, on each processor $j$, to form $\operatorname{Hist}_{j}(R)$ in time: $O\left(\min \left(\|H i s t(R)\|, \frac{\|R\|}{p}\right)\right)$.

Hence the creation of the histograms $\operatorname{Hist}_{i}(R)_{i=1, \ldots, p}$ of relation $R$ requires:

$$
O\left(\min \left(g *|H i s t(R)|+\|H i s t(R)\|, g * \frac{|R|}{p}+\frac{\|R\|}{p}\right)\right) .
$$

Similarly for relation $S$, the above technique is used to compute the fragments Hist $_{i}(S)_{i=1, \ldots, p}$ in time: $O\left(\min \left(g *|H i s t(S)|+\|H i s t(S)\|, g * \frac{|S|}{p}+\frac{\|S\|}{p}\right)\right)$.

Therefore, the creation of $\operatorname{Hist}_{i}(R)_{i=1, . ., p}$ and $\operatorname{Hist}_{i}(S)_{i=1, \ldots, p}$ requires:

$$
\begin{aligned}
\text { Time }_{\text {step.a }}=O & \left(\min \left(g *|\operatorname{Hist}(R)|+\|\operatorname{Hist}(R)\|, g * \frac{|R|}{p}+\frac{\|R\|}{p}\right)\right. \\
& \left.+\min \left(g *|\operatorname{Hist}(S)|+\|\operatorname{Hist}(S)\|, g * \frac{|S|}{p}+\frac{\|S\|}{p}\right)+l\right) .
\end{aligned}
$$

b. A stage of creation of the fragments $\operatorname{Hist}_{i}(R \bowtie S)_{i=1, \ldots, p}$ :

$\operatorname{Hist}_{i}(R \bowtie S)$ is computed, on each processor i, by intersecting $\operatorname{Hist}_{i}(R)$ and $\operatorname{Hist}_{i}(S)$ (i.e. by calculating $\left.\left\{v \mid v \in \operatorname{Hist}_{i}(R)\right\} \cap\left\{v \mid v \in \operatorname{Hist}_{i}(S)\right\}\right)$, in time:

$$
\text { Time }_{\text {step.b }}=O\left(\max _{i=1, . ., p}\left(\min \left(\left\|H i s t_{i}(R)\right\|,\left\|H i s t_{i}(S)\right\|\right)\right)\right) .
$$

c. We then compute, on each processor $j$, the intersections:

$$
\left\{\begin{array}{l}
\overline{H i s t}^{j}\left(R_{i}\right)=\operatorname{Hist}^{j}\left(R_{i}\right) \cap H_{i s t}(R \bowtie S) \text { for } i=1, . ., p \text { and } \\
\overline{H i s t}^{j}\left(S_{i}\right)=\operatorname{Hist}^{j}\left(S_{i}\right) \cap H i s t_{j}(R \bowtie S) \text { for } i=1, . ., p
\end{array}\right.
$$

in time: $O\left(\sum_{i}\left\|H i s t^{j}\left(R_{i}\right)\right\|+\sum_{i}\left\|H i s t^{j}\left(S_{i}\right)\right\|\right)$.

In accordance with stage a., one has: $\left\{\begin{array}{l}\sum_{i}\left\|\operatorname{Hist}^{j}\left(R_{i}\right)\right\| \leq \min \left(\|\operatorname{Hist}(R)\|, \frac{\|R\|}{p}\right) \\ \sum_{i}\left\|\operatorname{Hist}^{j}\left(S_{i}\right)\right\| \leq \min \left(\|\operatorname{Hist}(S)\|, \frac{\|S\|}{p}\right),\end{array}\right.$ and thus the total cost of this stage is:

$$
\text { Time }_{\text {step.c }}=O\left(\min \left(\|\operatorname{Hist}(R)\|, \frac{\|R\|}{p}\right)+\min \left(\|\operatorname{Hist}(S)\|, \frac{\|S\|}{p}\right)\right) .
$$

d. A stage of communication where each processor $i$ sends each fragment $\overline{H i s t}^{j}\left(R_{i}\right)$ to processor $j$ : During this stage of communication, each processor $i$, receives $\sum_{j}\left|\overline{H i s t}^{j}\left(R_{i}\right)\right|$ pages of data from the other processors in time 
$O\left(\sum_{j} g *\left|\overline{H i s t}^{j}\left(R_{i}\right)\right|+l\right)$. We point out that: Hist $\left(R_{i}\right)=\cup_{j} H i s t^{j}\left(R_{i}\right)$ and that: $\left|H i s t\left(R_{i}\right)\right|=\sum_{j}\left|H_{i s t}{ }^{j}\left(R_{i}\right)\right| \geq \sum_{j}\left|H_{i s t}{ }^{j}\left(R_{i}\right) \cap H i s t(R \bowtie S)\right|$

and thus $\left|H i s t\left(R_{i}\right)\right| \geq \sum_{j}\left|\overline{H i s t}^{j}\left(R_{i}\right)\right|$. Consequently the total cost of this stage is at most: Time $_{\text {step.d }}=O\left(g *\left|\overline{H i s t}\left(R_{i}\right)\right|+l\right)$.

Remark 2. $\cup_{j} \overline{H i s t}^{j}\left(R_{i}\right)$ is simply the intersection of Hist $\left(R_{i}\right)$ and Hist $(R \bowtie$ $S)$. This intersection is noted: $\overline{\operatorname{Hist}}\left(R_{i}\right)=\cup_{j} \overline{\operatorname{Hist}}^{j}\left(R_{i}\right)=\operatorname{Hist}\left(R_{i}\right) \cap \operatorname{Hist}(R \bowtie S)$.

e. A stage of the local semi-joins $R_{i} \ltimes S$ and $S_{i} \ltimes R$ computation:

Owing to the fact that, at the end of stage d., each processor $i$ holds the fragment $\overline{H i s t}\left(R_{i}\right)=\operatorname{Hist}\left(R_{i}\right) \cap \operatorname{Hist}(R \bowtie S)$, the semi-join $R_{i} \ltimes S$ (resp. $\left.S_{i} \ltimes R\right)$ can be computed, in parallel on each processor $i$, by a scan of the fragment $R_{i}$ (resp. $S_{i}$ ) and consulting the histogram $\overline{H i s t}\left(R_{i}\right)$ in time $O\left(\left\|R_{i}\right\|\right)$ (resp. $\left.O\left(\left\|S_{i}\right\|\right)\right)$. Thus, the total cost of this stage is: Time step.e $=O\left(\max _{i=1, . ., p}\left(\left\|R_{i}\right\|+\left\|S_{i}\right\|\right)\right)$.

The total cost of the two semi-joins $R \ltimes S$ and $S \ltimes R$ computation, is thus the sum of the above five stages:

$$
\begin{aligned}
\text { Time }_{s j}= & O\left(\min \left(g *|\operatorname{Hist}(R)|+\|\operatorname{Hist}(R)\|, g * \frac{|R|}{p}+\frac{\|R\|}{p}\right)+\max _{i=1, \ldots, p}\left\|R_{i}\right\|\right. \\
& \left.+\min \left(g *|\operatorname{Hist}(S)|+\|\operatorname{Hist}(S)\|, g * \frac{|S|}{p}+\frac{\|S\|}{p}\right)+\max _{i=1, . ., p}\left\|S_{i}\right\|+l\right) .
\end{aligned}
$$

Using the previous results, the following proposition gives an upper bound the global BSP cost for semi-join computation.

Proposition 3. Let $R_{i=1, \ldots, p}$ and $S_{i=1, \ldots, p}$ be the fragments of $R$ and $S$ on $p$ processors. The semi-joins $R \ltimes S$ and $S \ltimes R$ can be carried out in time:

$$
\begin{aligned}
\text { Time }_{\text {semi-joins }}=O( & c_{i / o} * \max _{i=1, \ldots, p}\left(\left|R_{i}\right|+\left|S_{i}\right|\right)+\max _{i=1, \ldots, p}\left\|R_{i}\right\|+\max _{i=1, \ldots, p}\left\|S_{i}\right\|+l \\
& +\min \left(g *|\operatorname{Hist}(R)|+\|\operatorname{Hist}(R)\|, g * \frac{|R|}{p}+\frac{\|R\|}{p}\right) \\
& \left.+\min \left(g *|\operatorname{Hist}(S)|+\|\operatorname{Hist}(S)\|, g * \frac{|S|}{p}+\frac{\|S\|}{p}\right)\right),
\end{aligned}
$$

where $c_{i} / o$ is the cost to read/write a page of data from disk.

Proof: Starting from the local fragments $R_{i=1, \ldots, p}$ (resp. $S_{i=1, \ldots, p}$ ) of relation $R$ (resp. $S)$, the local histograms $\operatorname{Hist}\left(R_{i}\right)_{i=1, \ldots, p}\left(\operatorname{resp} . \operatorname{Hist}\left(S_{i}\right)_{i=1, \ldots, p}\right)$ can be created in parallel by a scan of $R_{i}$ (resp. $S_{i}$ ) on processor $i$ in time: $c_{i / o} *$ $\max _{i=1, . ., p}\left|R_{i}\right|$ (resp. $\left.c_{i / o} * \max _{i=1, . ., p}\left|S_{i}\right|\right)$. Hence this step costs:

$$
\text { Time }_{s j_{a}}=O\left(c_{i / o} * \max _{i=1, . ., p}\left(\left|R_{i}\right|+\left|S_{i}\right|\right)\right)
$$

At the end of this step, each processor $i$ holds $H i s t\left(R_{i}\right)$ and $H i s t\left(S_{i}\right)$. 
By using Proposition 2 and by using an appropriate hashing function (see remark 1), the two semi-joins, $R \ltimes S$ and $S \ltimes R$ are computed in time:

$$
\begin{aligned}
\text { Time }_{s j_{b}}= & O\left(\min \left(g *|\operatorname{Hist}(R)|+\|\operatorname{Hist}(R)\|, g * \frac{|R|}{p}+\frac{\|R\|}{p}\right)+\max _{i=1, \ldots, p}\left\|R_{i}\right\|\right. \\
& \left.+\min \left(g *|\operatorname{Hist}(S)|+\|\operatorname{Hist}(S)\|, g * \frac{|S|}{p}+\frac{\|S\|}{p}\right)+\max _{i=1, \ldots, p}\left\|S_{i}\right\|+l\right) .
\end{aligned}
$$

The semi-joins computation has the sum of the above two costs, which completes the proof of the proposition.

Remark 3. Sequential semi-joins $R \ltimes S$ and $S \ltimes S$ require at least the time to scan input relations $R$ and $S$ : bound $_{\text {in }_{1}}=\Omega\left(c_{i / o} *|R|+\|R\|+c_{i / o} *|S|+\|S\|\right)$. Parallel processing using p processors requires therefore bound inf $_{p}=\frac{1}{p} *$ bound $_{\text {inf } f_{1}}$.

We recall that, in all existing parallel semi-join algorithms, semi-joins are performed in two phases: a redistribution phase (generally based on hashing functions) according to the values of the semi-join attribute of input relations and then a sequential scan of local relations. In these algorithms, the redistribution phase is very sensitive to data skew $[2,3,1]$.

Using our approach, the semi-join computation requires only the distribution of histograms which make it insensitive to data skew. The complexity of our algorithm is asymptotically optimal owing to the fact that all the terms of the global BSP cost are of the same order of those of bound inf $_{p}$.

\section{Conclusion}

In this paper, we have introduced the first parallel semi-join algorithm allowing to reduce the communication costs to the minimum owing to the fact that only histograms are redistributed across the network and the sizes of these histograms are very small compared to the sizes of input relations.

Using the BSP cost model, the algorithm is proved to have an asymptotic optimal complexity while guaranteeing perfect balancing properties even in the presence of highly skewed data. This algorithm can be used efficiently to optimize the execution time of complex queries by reducing the amount of input data for each sub-query and the communication costs.

Future work will consist in studying how histograms could be used to generate efficient query execution plans for complex queries. We also plan to study how these histograms could help to optimize resource allocation in distributed architectures.

\section{References}

1. M. Bamha and M. Exbrayat. Pipelining a skew-insensitive parallel join algorithm. Parallel Processing Letters, Volume 3, Number 3, pages 317-328, 2003. 
2. M. Bamha and G. Hains. A skew insensitive algorithm for join and multi-join operation on Shared Nothing machines. In the 11th International Conference on Database and Expert Systems Applications DEXA'2000, volume 1873, 2000.

3. M. Bamha and G. Hains. A frequency adaptive join algorithm for Shared Nothing machines. Journal of Parallel and Distributed Computing Practices (PDCP), Volume 3, Number 3, pages 333-345, September 1999.

4. J. Lawrence Carter and Mark N. Wegman. Universal classes of hash functions. Journal of Computer and System Sciences, 18(2):143-154, April 1979.

5. D. J. DeWitt, J. F. Naughton, D. A. Schneider, and S. Seshadri. Practical Skew Handling in Parallel Joins. In Proceedings of the 18th VLDB Conference, pages 27-40, Vancouver, British Columbia, Canada, 1992.

6. K. A. Hua and C. Lee. Handling data skew in multiprocessor database computers using partition tuning. Proc. of the 17th International Conference on Very Large Data Bases, pages 525-535, Barcelona, Catalonia, Spain, 1991.

7. M. Kitsuregawa and Y. Ogawa. Bucket spreading parallel hash: A new, robust, parallel hash join method for skew in the super database computer (SDC). 16th International Conference on Very Large Data Bases, pages 210-221, 1990.

8. M. Seetha and P. S. Yu. Effectiveness of parallel joins. IEEE, Transactions on Knowledge and Data Enginneerings, 2(4):410-424, December 1990.

9. D. B. Skillicorn, J. M. D. Hill, and W. F. McColl. Questions and Answers about BSP. Scientific Programming, 6(3):249-274, 1997.

10. Konrad Stocker, Donald Kossmann, Reinhard Braumandl, and Alfons Kemper. Integrating semi-join-reducers into state-of-the-art query processors. In Proceedings of the 17th International Conference on Data Engineering, pages 575 - 584, 2001.

11. Joel L. Wolf, Daniel M. Dias, Philip S. Yu, and John Turek. New algorithms for parallelizing relational database joins in the presence of data skew. IEEE Transactions on Knowledge and Data Engineering, 6(6):990-997, 1994. 